\title{
Existence of Solution for Double-Phase Problem with Singular Weights
}

\author{
Zhi-Yuan Chen, ${ }^{1}$ Bin Ge $\mathbb{D}^{2},{ }^{2}$ Wen-Shuo Yuan, ${ }^{2}$ and Xiao-Feng Cao ${ }^{2}$ \\ ${ }^{1}$ College of Computer Science and Technology, Harbin Engineering University, Harbin, 150001, P.R., China \\ ${ }^{2}$ College of Mathematical Sciences, Harbin Engineering University, Harbin, 150001, P.R., China
}

Correspondence should be addressed to Bin Ge; gebin04523080261@163.com

Received 12 May 2020; Revised 15 June 2020; Accepted 25 June 2020; Published 15 July 2020

Academic Editor: Carlo Bianca

Copyright ( 2020 Zhi-Yuan Chen et al. This is an open access article distributed under the Creative Commons Attribution License, which permits unrestricted use, distribution, and reproduction in any medium, provided the original work is properly cited.

\begin{abstract}
The aim of this paper is to establish the existence of solutions for singular double-phase problems depending on one parameter. This work improves and complements the existing ones in the literature. There seems to be no results on the existence of solutions for singular double-phase problems.
\end{abstract}

\section{Introduction and Main Results}

The study of various mathematical problems involving the double-phase operator has become very attractive in recent decades. The existence and multiplicity of solutions of double-phase Dirichlet problems has been studied by several authors (see, e.g., [1-8]); in particular, for the eigenvalues of the double-phase operator, see [7]. For other double-phase problems with variable exponents, there are the works of Zhang and Radulescu [9], Shi et al. [10], and Cencelj et al. [11].
But up to now, to the best of our knowledge, no paper discussing the existence of solutions for singular double-phase problems via critical point theory can be found in the existing literature. In order to fill in this gap, we study double-phase problems from a more extensive viewpoint. More precisely, we are going to prove that problem $\left(P_{\lambda}\right)$ has at least one solution. To the best of our knowledge, this is one of the first works which combines a singular term and indefinite term in one problem.

This paper is concerned with the existence of solutions to the following singular double-phase problem:

$$
\begin{cases}-\operatorname{div}\left(|\nabla u|^{p-2} \nabla u+a(x)|\nabla u|^{q-2} \nabla u\right)=b(x)|u|^{-\theta-1} u+\lambda f(x, u), & \text { in } \Omega, \\ u=0, & \text { on } \partial \Omega,\end{cases}
$$

where $\Omega$ is a smooth bounded domain in $\mathbb{R}^{N}, N \geq 2,0<\theta<1$, $\lambda \in \mathbb{R}, a: \bar{\Omega} \mapsto[0,+\infty)$ is Lipschitz continuous, and $b$ is a given measurable function. The precise conditions on the data will be presented later.

Problems of the above type arise for instance in nonlinear elasticity. The main reasons are to describe the behavior of Lavrentiev's phenomenon; we refer to [12-14]. In fact, Zhikov intended to provide models for strongly anisotropic materials in the context of homogenization. In particular, he considered the following functional:

$$
\int_{\Omega}\left(|\nabla u|^{p}+a(x)|\nabla u|^{q}\right) d x, \quad 0 \leq a(x) \leq L, 1<p<q
$$

where the modulating coefficient $a(x)$ dictates the geometry of the composite made of two differential materials, with 
hardening exponents $p$ and $q$, respectively. Recently, there is a wide literature on the regularity theory for minimizers of variational problems and solutions of differential equations with the double-phase operator; far from being complete, we refer the readers to [15-21], respectively, and references therein.

In the entire paper, we suppose the following assumptions:

$H(a): a: \bar{\Omega} \mapsto[0,+\infty)$ is Lipschitz continuous and $1<$ $p<q<N$ are chosen such that $(q / p)<1+(1 / N)$.

$H(b): b \in L^{p /(p+\theta-1)}(\Omega)$ such that $b(x)>0$ in $\Omega$.

$H(f)_{1}: f: \Omega \times \mathbb{R} \longrightarrow \mathbb{R}$ is a continuous function such that for a.a. $x \in \Omega, f(x, 0)=0$, and

(i) there exists positive measurable subsets $\Omega_{1} \subset \Omega$ and $c \in L^{s_{1}}(\Omega)$ such that $c(x) \geq 0$ on $\Omega_{1}$, and

$$
\lim _{t \rightarrow 0} \frac{f(x, t)}{c(x)|t|^{r_{1}-2} t}=0 \quad \text { uniformly for a.a. } x \in \Omega \text {, }
$$

where $1<r_{1}<p<N<s_{1}$;

(ii) there exists $d \in L^{s_{2}}(\Omega)$ such that $d(x)>0$ on $\Omega$, and

$$
\lim _{t \rightarrow+\infty} \frac{f(x, t)}{d(x)|t|^{r_{2}-2} t}=0 \quad \text { uniformly for a.a. } x \in \Omega \text {, }
$$

where $1<r_{2}<p<N<s_{2}$;

(iii) there exists $M>0$ such that

$$
\int_{\Omega} F(x, t) d x>0, \quad \forall|t|>M
$$

where $F(x, t)=\int_{0}^{t} f(x, s) d s$.

Example 1. The following function satisfies hypotheses $H(f)_{1}$ :

$$
f(x, t)= \begin{cases}c(x)\left(|t|^{p-2} t-|t|^{q-2} t\right), & \text { if }|t| \leq 1, \\ d(x)\left(|t|^{\alpha-2} t-\frac{t}{|t|}\right), & \text { if }|t| \geq 1,\end{cases}
$$

with $1<\alpha<r_{2}$.

We are now in the position to state our main results. Firstly, problem $\left(P_{\lambda}\right)$ has a solution when $\lambda \leq 0$.

Theorem 1. Assume that $H(a), H(b)$, and $H(f)_{1}$ hold. Then for all $\lambda \leq 0$, problem $\left(P_{\lambda}\right)$ has at least one nontrivial weak solution with negative energy.

Moreover, we also show that problem $\left(P_{\lambda}\right)$ has a solution when $\lambda>0$. In order to do this task, the following conditions are needed:

$H(f)_{2}: f: \Omega \times \mathbb{R} \longrightarrow \mathbb{R}$ is a Carathéodory function such that for a.a. $x \in \Omega, f(x, 0)=0$, and (i) there exists $C>0$ and $d \in L^{s_{2}}(\Omega)$ such that

$$
f(x, t) \leq C d(x)|t|^{r_{2}-2} t, \quad \forall(x, t) \in \Omega \times \mathbb{R},
$$

where $1<r_{2}<p<s_{2}$;

(ii) there exists a positive measurable subset $\Omega_{1} \subset \Omega$ such that

$$
f(x, t), \quad d(x)>0, \forall x \in \Omega_{1}, \forall t>0 .
$$

Example 2. The following functions satisfy hypotheses $H(f)_{2}$ :

$$
\begin{aligned}
& f_{1}(x, t)=C d(x)|t|^{r_{2}-2} t, \\
& f_{2}(x, t)=\left\{\begin{array}{l}
C d(x) t^{p-1}, \quad \text { if } t \in[0,1], \\
C d(x) t^{r_{2}-1}, \quad \text { if } t \in[1,+\infty), \\
-C d(x)(-t)^{r_{2}-1}, \quad \text { if } t \in[-1,0], \\
-C d(x)(-t)^{p-1}, \quad \text { if } t \in(-\infty,-1] .
\end{array}\right.
\end{aligned}
$$

Theorem 2. Assume that $H(a), H(b)$, and $H(f)_{2}$ hold. Then for all $\lambda \geq 0$, problem $\left(P_{\lambda}\right)$ has at least one nontrivial weak solution with negative energy.

The rest of this paper is organized as follows. In Section 2, we present some necessary preliminary knowledge on space $W_{0}^{1, H}(\Omega)$. In Section 3, the proof of the main results is given.

\section{Preliminaries}

In order to discuss problem $(P)$, we need some facts on space $W_{0}^{1, H}(\Omega)$ which are called Musielak-Orlicz-Sobolev spaces. For this reason, we will recall some properties involving the Musielak-Orlicz spaces, which can be found in [7, 22-24] and references therein.

Denote by $N(\Omega)$ the set of all generalized $N$-function. For $1<p<q$ and $0 \leq a(\cdot) \in L^{1}(\Omega)$, we define $H(x, t)=t^{p}+a(x)$ $t^{q}, \forall(x, t) \in \Omega \times[0,+\infty)$. It is clear that $H \in N(\Omega)$ is a locally integrable function and $H(x, 2 t) \leq 2^{q} H(x, t), \forall(x, t) \in \Omega \times[0$, $+\infty)$ which is called condition $\left(\Delta_{2}\right)$.

The Musielak-Orlicz space $L^{H}(\Omega)$ is defined by

$L^{H}(\Omega)=\left\{u: \Omega \longrightarrow \mathbb{R}\right.$ measurable $\left.: \int_{\Omega} H(x,|u|) d x<+\infty\right\}$,

endowed with the Luxemburg norm $|u|_{H}=\inf \left\{\lambda>0: \int_{\Omega}\right.$ $H(x,|u / \lambda|) d x \leq 1\}$. The Musielak-Orlicz-Sobolev space $W^{1, H}(\Omega)$ is defined by

$$
W^{1, H}(\Omega)=\left\{u \in L^{H}(\Omega):|\nabla u| \in L^{H}(\Omega)\right\},
$$

and it is equipped with the norm $\|u\|=|u|_{H}+|\nabla u|_{H}$. We denote by $W_{0}^{1, H}(\Omega)$ the completion of $C_{0}^{\infty}(\Omega)$ in $W^{1, H}(\Omega)$. With these norms, the spaces $L^{H}(\Omega), W_{0}^{1, H}(\Omega)$, and $W^{1, H}$ 
$(\Omega)$ are separable reflexive Banach spaces (see [7] for the details).

Proposition 3. ([1], Proposition 2.1). have

Set $\rho_{H}(u)=\int_{\Omega}\left(|u|^{p}+a(x)|u|^{q}\right) d x$. For $u \in L^{H}(\Omega)$, we

(i) For $u \neq 0,|u|_{H}=\lambda \Leftrightarrow \rho_{H}(u / \lambda)=1$

(ii) $|u|_{H}<1(=1 ;>1) \Leftrightarrow \rho_{H}(u)<1(=1 ;>1)$

(iii) If $|u|_{H} \geq 1$, then $|u|_{H}^{p} \leq \rho_{H}(u) \leq|u|_{H}^{q}$

(iv) If $|u|_{H} \leq 1$, then $|u|_{H}^{q} \leq \rho_{H}(u) \leq|u|_{H}^{p}$

Proposition 4. ([7], Proposition 2.15, Proposition 2.18).

(1) If $1 \leq s \leq N p /(N-p)$, then the embedding from $W_{0}^{1, H}$ $(\Omega)$ to $L^{s}(\Omega)$ is continuous. In particular, if $s \in[1, N$ $p /(N-p))$, then the embedding $W_{0}^{1, H}(\Omega) \hookrightarrow L^{s}(\Omega)$ is compact

(2) Assume that $H(a)$ holds. Then, Poincare's inequality holds; that is, there exists a positive constant $C_{0}$ such that $|u|_{H} \leq C_{0}\|u\|, \forall u \in W_{0}^{1, H}(\Omega)$

By the above Proposition, there exists $c_{\tau}>0$ such that $|u|_{\tau} \leq c_{\tau}\|u\|, \forall u \in W_{0}^{H}(\Omega)$, where $|u|_{\tau}$ denotes the usual norm in $L^{\tau}(\Omega)$ for all $1 \leq \tau<N p /(N-p)$. It follows from (2) of Proposition 4 that $|\nabla u|_{H}$ is an equivalent norm in $W_{0}^{1, H}(\Omega)$. We will use the equivalent norm in the following discussion and write $\|u\|=|\nabla u|_{H}$ for simplicity.

In order to discuss the problem $(P)$, we need to define a functional in $W_{0}^{1, H}(\Omega)$ :

$$
J(u)=\int_{\Omega}\left(\frac{1}{p}|\nabla u|^{p}+\frac{a(x)}{q}|\nabla u|^{q}\right) d x .
$$

We know that (see [25], P63, example) $J \in C^{1}\left(W_{0}^{1, H}(\Omega)\right.$, $\mathbb{R})$ and the double-phase operator

$$
-\operatorname{div}\left(|\nabla u|^{p-2} \nabla u+a(x)|\nabla u|^{q-2} \nabla u\right)
$$

is the derivative operator of $J$ in the weak sense. Moreover, similar to the proof of Theorem 3.1 in [25], we know that the energy functional $J$ is sequentially weakly lower semicontinuous.

\section{Variational Setting and Proof of the Main Results}

For any $\lambda \in \mathbb{R}$ and each $u \in E$, we define

$$
\varphi_{\lambda}(u)=J(u)-\int_{\Omega} \frac{b(x)}{1-\theta}|u|^{1-\theta} d x-\lambda \int_{\Omega} F(x, u) d x
$$

where $J(u)=\int_{\Omega}\left((1 / p)|\nabla u|^{p}+(a(x) / q)|\nabla u|^{q}\right) d x$. By using $H(f)_{1}$, we get $r_{1}<s_{1} r_{1} /\left(s_{1}-1\right)<N p /(N-p)$ and $r_{2}<s_{2} r_{2} /$ $\left(s_{2}-1\right)<N p /(N-p)$. Also, by Proposition 4 (1) we deduce that embeddings $E \hookrightarrow L^{s_{1} r_{1} /\left(s_{1}-1\right)}(\Omega)$ and $E \hookrightarrow L^{s_{2} r_{2} /\left(s_{2}-1\right)}(\Omega)$ are compact and continuous. Furthermore, there exists a constant $C_{0}>0$ such that

$$
\max \left\{|u|_{s_{1} r_{1} /\left(s_{1}-1\right)},|u|_{p},|u|_{s_{2} r_{2} /\left(s_{2}-1\right)}\right\} \leq C_{0}\|u\|, \quad \forall u \in E
$$

Now, we are ready to prove Theorem 1 .

Proof of Theorem 1. To complete the proof of the main result, we need to consider the following three steps.

Step 1 . We first show that for every $\lambda \leq 0$, the functional $\varphi_{\lambda}$ is coercive on $E$.

Let $\lambda \leq 0$ be fixed. Put $\Omega_{M}=\bar{\Omega} \times[-M, M]$. Clearly, from the continuity of $f$, there exists $K_{M}>0$ such that

$$
K_{M}=\max _{(x, t) \in \Omega_{M}}|f(x, t)|
$$

Thus, we deduce that for any $x \in \Omega$ and $|t| \leq M$,

$$
|F(x, t)|=\left|\int_{0}^{t} f(x, s) d s\right| \leq \int_{0}^{t}|f(x, s)| d s \leq M K_{M} .
$$

By virtue of assumption $H(f)_{1}$ (iii), (15), (17), and Proposition 3 , one has for any $u \in E$ with $\|u\|>1$

$$
\begin{aligned}
\varphi_{\lambda}(u) \geq & \frac{1}{q}\|u\|^{p}-\left.\left.\frac{1}{1-\theta}|b|_{p /(p+\theta-1)}|| u\right|^{1-\theta}\right|_{p /(1-\theta)} \\
& -\lambda \int_{|u(x)| \leq M} F(x, u) d x-\lambda \int_{|u(x)|>M} F(x, u) d x \\
\geq & \frac{1}{q}\|u\|^{p}-\frac{1}{1-\theta}|b|_{p /(p+\theta-1)}|u|_{p}^{1-\theta}+\lambda M K_{M}|\Omega| \\
\geq & \frac{1}{q}\|u\|^{p}-\frac{1}{1-\theta}|b|_{p /(p+\theta-1)} C_{0}^{1-\theta}\|u\|^{1-\theta}+\lambda M K_{M}|\Omega| .
\end{aligned}
$$

Since $0<\theta<1$ and $1-\theta<p$, so this implies $\varphi_{\lambda}(u) \longrightarrow$ $+\infty$ as $\|u\| \longrightarrow+\infty$. The proof of Step 1 is now completed.

Step 2. We show that there exists $\xi \in E$ with $\xi>0, \varphi_{\lambda}(t \xi)<0$ for $t>0$ small enough.

Let $\xi \in C_{0}^{\infty}(\Omega)$ such that supp $(\xi) \subset \Omega_{1} \subset \Omega, \xi=1$ in a subset $\Omega_{0} \subset \operatorname{supp}(\xi)$, and $0 \leq \xi \leq 1$ in $\Omega_{1}$. Thus, by condition $H(f)_{1}(\mathrm{i})$, it follows that there exists $t_{0} \in(0,1)$ such that

$F(x, t \xi(x)) \leq \frac{c(x)}{r_{1}}|t \xi(x)|^{r_{1}} \leq c(x)|t \xi(x)|^{r_{1}}, \quad \forall t \in\left(0, t_{0}\right), x \in \Omega$. 
Hence, for any $t \in\left(0, t_{0}\right)$, from $H(b)$ and $H(f)_{1}$ (i), we deduce that

$$
\begin{aligned}
\varphi_{\lambda}(t \xi) \leq & \frac{t^{p}}{p} \max \left\{\|\xi\|^{p},\|\xi\|^{q}\right\} \\
& -\frac{t^{1-\theta}}{1-\theta} \int_{\Omega} b(x)|\xi|^{1-\theta} d x-\lambda \int_{\Omega} F(x, t \xi) d x \\
\leq & \frac{t^{p}}{p} \max \left\{\|\xi\|^{p},\|\xi\|^{q}\right\}-\frac{1}{1-\theta} \int_{\Omega} b(x)|t \xi|^{1-\theta} d x \\
& -\lambda|t|^{r_{1}} \int_{\Omega_{1}} c(x)|\xi|^{r_{1}} d x \\
\leq & t^{r_{1}}\left[\max \left\{\|\xi\|^{p},\|\xi\|^{q}\right\}-\lambda \int_{\Omega_{1}} c(x)|\xi|^{r_{1}} d x\right] \\
& -\frac{t^{1-\theta}}{1-\theta} \int_{\Omega} b(x)|\xi|^{1-\theta} d x .
\end{aligned}
$$

Since $r_{1}>1-\theta$, we have $\varphi_{\lambda}(t \xi)<0$ for $t<t_{1}$ with

$0<t_{1}<\min \left\{t_{0},\left(\frac{(1 /(1-\theta)) \int_{\Omega} b(x)|\xi|^{1-\theta} d x}{\max \left\{\|\xi\|^{p},\|\xi\|^{q}\right\}-\lambda \int_{\Omega_{1}} c(x)|\xi|^{r_{1}} d x}\right)^{1 /\left(r_{1}+\theta-1\right)}\right\}$.

The proof of Step 2 is now complete.

Step 3 . We show that there exists $u_{\lambda} \in E$ such that $\varphi_{\lambda}\left(u_{\lambda}\right)=$ $\inf _{u \in E} \varphi_{\lambda}(u)$ for any $\lambda \leq 0$.

Let $\left\{u_{\lambda}^{n}\right\} \subset E$ be a minimizing sequence of $\varphi_{\lambda}$. Then, using Step 1 , we get that $\left\{u_{\lambda}^{n}\right\}$ is a bounded sequence. So, there exists $u_{\lambda} \in E$ such that, up to a subsequence,

$$
\begin{gathered}
u_{\lambda}^{n} \rightarrow u_{\lambda} \quad \text { in } E, \\
u_{\lambda}^{n} \longrightarrow u_{\lambda} \quad \text { in } L^{p}(\Omega), \\
u_{\lambda}^{n}(x) \rightarrow u_{\lambda}(x) \quad \text { a.e.in } \Omega .
\end{gathered}
$$

Recall that $J$ is sequentially weakly lower semicontinuous, and so we deduce that

$$
J\left(u_{\lambda}\right) \leq \liminf _{n \rightarrow+\infty} J\left(u_{\lambda}^{n}\right) .
$$

Now, using Hölder's inequality, we get that, as $n \longrightarrow+\infty$,

$$
\begin{aligned}
\int_{\Omega} b(x)\left|u_{\lambda}^{n}\right|^{1-\theta} d x \leq & \int_{\Omega} b(x)\left|u_{\lambda}\right|^{1-\theta} d x+\int_{\Omega} b(x)\left|u_{\lambda}^{n}-u_{\lambda}\right|^{1-\theta} d x \\
& \leq \int_{\Omega} b(x)\left|u_{\lambda}\right|^{1-\theta} d x \\
& +|b|_{p /(p+\theta-1)}|| u_{\lambda}^{n}-\left.\left.u_{\lambda}\right|^{1-\theta}\right|_{p /(1-\theta)}
\end{aligned}
$$

$$
\begin{aligned}
& =\int_{\Omega} b(x)\left|u_{\lambda}\right|^{1-\theta} d x+|b|_{p /(p+\theta-1)}\left|u_{\lambda}^{n}-u_{\lambda}\right|_{p}^{1-\theta} \\
& =\int_{\Omega} b(x)\left|u_{\lambda}\right|^{1-\theta} d x+o_{n}(1) .
\end{aligned}
$$

Analogously,

$$
\begin{aligned}
\int_{\Omega} b(x)\left|u_{\lambda}\right|^{1-\theta} d x \leq & \int_{\Omega} b(x)\left|u_{\lambda}^{n}\right|^{1-\theta} d x+\int_{\Omega} b(x)\left|u_{\lambda}-u_{\lambda}^{n}\right|^{1-\theta} d x \\
\leq & \int_{\Omega} b(x)\left|u_{\lambda}^{n}\right|^{1-\theta} d x \\
& +\left.|b|_{p /(p+\theta-1)}\left|u_{\lambda}^{n}-u_{\lambda}\right|^{1-\theta}\right|_{p /(1-\theta)} \\
= & \int_{\Omega} b(x)\left|u_{\lambda}^{n}\right|^{1-\theta} d x+|b|_{p /(p+\theta-1)}\left|u_{\lambda}^{n}-u_{\lambda}\right|_{p}^{1-\theta} \\
= & \int_{\Omega} b(x)\left|u_{\lambda}^{n}\right|^{1-\theta} d x+o_{n}(1) .
\end{aligned}
$$

Hence, by (24) and (25), one yields

$$
\lim _{n \rightarrow+\infty} \int_{\Omega} b(x)\left|u_{\lambda}^{n}\right|^{1-\theta} d x=\int_{\Omega} b(x)\left|u_{\lambda}\right|^{1-\theta} d x
$$

Moreover, using assumptions $H(f)_{1}$ (i) and $H(f)_{1}$ (ii), for all $\varepsilon>0$, there exists $C_{\varepsilon}>0$ such that

$$
\left|F\left(x, u_{\lambda}\right)\right| \leq \varepsilon c(x)\left|u_{\lambda}\right|^{r_{1}}+C_{\varepsilon} d(x)\left|u_{\lambda}\right|^{r_{2}} .
$$

The above information and Hölder's inequality imply

$$
\begin{aligned}
\int_{\Omega}\left|F\left(x, u_{\lambda}\right)\right| d x & \leq\left.\left.\varepsilon|C|_{s_{1}}|| u_{\lambda}\right|^{r_{1}}\right|_{s_{1} /\left(s_{1}-1\right)}+\left.\left.C_{\varepsilon}|d|_{s_{2}}|| u_{\lambda}\right|^{r_{2}}\right|_{s_{2} /\left(s_{2}-1\right)} d \\
& =\varepsilon|C|_{s_{1}}\left|u_{\lambda}\right|_{s_{1} r_{1} /\left(s_{1}-1\right)}^{r_{1}}+C_{\varepsilon}|d|_{s_{2}}\left|u_{\lambda}\right|_{s_{2} r_{2} /\left(s_{2}-1\right)}^{r_{2}} .
\end{aligned}
$$

Again, by Proposition 4 (1), we deduce that

$$
\begin{gathered}
E \hookrightarrow L^{s_{1} r_{1} /\left(s_{1}-1\right)}(\Omega), \quad u_{\lambda}^{n} \longrightarrow u \operatorname{in} L^{s_{1} r_{1} /\left(s_{1}-1\right)}(\Omega), \\
E \hookrightarrow L^{s_{2} r_{2} /\left(s_{2}-1\right)}(\Omega), \quad u_{\lambda}^{n} \longrightarrow u \operatorname{in} L^{s_{2} r_{2} /\left(s_{2}-1\right)}(\Omega), \\
u_{\lambda}^{n} \longrightarrow u \quad \text { for a.a. } x \in \Omega, \\
F\left(x, u_{\lambda}^{n}(x)\right) \longrightarrow F\left(x, u_{\lambda}(x)\right) \quad \text { for a.a. } x \in \Omega .
\end{gathered}
$$

Thus, using the fact that $\left\{u_{\lambda}^{n}\right\}$ is bounded in $E$ and the dominated convergence theorem, we can infer that

$$
\lim _{n \rightarrow+\infty} \int_{\Omega} F\left(x, u_{\lambda}^{n}(x)\right)=\int_{\Omega} F\left(x, u_{\lambda}(x)\right) d x .
$$


Hence, for every $\lambda<0$, by (26) and (30), one yields

$$
\begin{aligned}
\liminf _{n \rightarrow \infty} \varphi_{\lambda}\left(u_{\lambda}^{n}\right)= & \liminf _{n \rightarrow \infty}\left(J\left(u_{\lambda}^{n}\right)-\int_{\Omega} \frac{b(x)}{1-\theta}\left|u_{\lambda}^{n}\right|^{1-\theta} d x\right. \\
& \left.-\lambda \int_{\Omega} F\left(x, u_{\lambda}^{n}\right) d x\right) \\
= & \liminf _{n \rightarrow \infty} J\left(u_{\lambda}^{n}\right)-\lim _{n \rightarrow \infty} \int_{\Omega} \frac{b(x)}{1-\theta}\left|u_{\lambda}^{n}\right|^{1-\theta} d x \\
& -\lambda \lim _{n \rightarrow \infty} \int_{\Omega} F\left(x, u_{\lambda}^{n}\right) d x \\
\geq & J\left(u_{\lambda}\right)-\int_{\Omega} \frac{b(x)}{1-\theta}\left|u_{\lambda}\right|^{1-\theta} d x-\lambda \int_{\Omega} F\left(x, u_{\lambda}\right) d x \\
= & J\left(u_{\lambda}\right)-\Phi\left(u_{\lambda}\right)-\lambda \Psi\left(u_{\lambda}\right)=\varphi_{\lambda}\left(u_{\lambda}\right),
\end{aligned}
$$

which implies that $\varphi_{\lambda}$ is weakly lower semicontinuous, and consequently,

$$
\varphi_{\lambda}\left(u_{\lambda}\right) \leq \liminf _{u \in E} \varphi_{\lambda}\left(u_{\lambda}^{n}\right)=\inf _{v \in E} \varphi_{\lambda}(v) \leq \varphi_{\lambda}\left(u_{\lambda}\right)
$$

which implies that

$$
\varphi\left(u_{\lambda}\right)=\inf _{u \in E} \varphi_{\lambda}\left(u_{\lambda}^{n}\right)
$$

So, we complete Step 3.

Therefore, combining the above Steps 2 and 3, we deduce that $u_{\lambda}$ is the required nontrivial solution of problem $\left(P_{\lambda}\right)$. Therefore, we complete the Proof of Theorem 1.

Now, we are ready to prove Theorem 2 .

Proof of Theorem 2. To complete the proof of the main result, we need to consider the following three steps.

Step 1. We first show that for every $\lambda \geq 0$, the functional $\varphi_{\lambda}$ is coercive on $E$.

Firstly, due to condition $H(f)_{2}$ (i), one has

$$
F(x, t) \leq \frac{C d(x)}{r_{2}}|t|^{r_{2}}, \quad \forall(x, t) \in \Omega \times \mathbb{R} .
$$

Again, using the condition $H(f)_{2}$ (i), Hölder's inequality, Proposition 3, and relation (34), we deduce that for any $u \in E$ with $\|u\|>1$, the following inequality holds true:

$$
\begin{aligned}
\varphi_{\lambda}(u) \geq & \frac{1}{q}\|u\|^{p}-\left.\left.\frac{1}{1-\theta}|b|_{p /(p+\theta-1)}|| u\right|^{1-\theta}\right|_{p /(1-\theta)} \\
& -\frac{\lambda C}{r_{2}} \int_{\Omega}|d(x)||u|^{r_{2}} d x
\end{aligned}
$$

$$
\begin{aligned}
\geq & \frac{1}{q}\|u\|^{p}-\frac{1}{1-\theta}|b|_{p /(p+\theta-1)}|u|_{p}^{1-\theta} \\
& -\left.\left.\frac{\lambda C}{r_{2}}|d|_{s_{2}}|| u\right|^{r_{2}}\right|_{s_{2} /\left(s_{2}-1\right)} \\
= & \frac{1}{q}\|u\|^{p}-\frac{1}{1-\theta}|b|_{p /(p+\theta-1)}|u|_{p}^{1-\theta} \\
& -\frac{\lambda C C_{0}^{r_{2}}}{r_{2}}|d|_{s_{2}}\|u\|^{r_{2}} .
\end{aligned}
$$

Since $1-\theta<1<r_{2}<p$, we infer that $\varphi_{\lambda}(u) \longrightarrow+\infty$ as $\|u\| \longrightarrow+\infty$. The proof of Step 1 is now complete.

Step 2. We show that there exists $\xi \in E$ with $\xi>0, \varphi_{\lambda}(t \xi)<0$ for $t>0$ small enough.

Let $\xi \in C_{0}^{\infty}(\Omega)$ such that $\operatorname{supp}(\xi) \subset \Omega_{1} \subset \Omega, \xi=1$ in a subset $\Omega_{0} \subset \operatorname{supp}(\xi)$, and $0 \leq \xi \leq 1$ in $\Omega_{1}$. Thus, by condition $H(f)_{2}$ (ii), it follows that

$$
F(x, t \xi(x)) \geq 0, \quad \forall t \in(0,1), x \in \Omega .
$$

Hence, for any $t \in(0,1)$, from $H(b)$ and $H(f)_{2}$ (ii), we deduce that

$$
\begin{aligned}
\varphi_{\lambda}(t \xi)= & \int_{\Omega}\left(\frac{1}{p}|\nabla u|^{p}+\frac{a(x)}{q}|\nabla u|^{q}\right) d x \\
& -\int_{\Omega} \frac{b(x)}{1-\theta}|t \xi|^{1-\theta} d x-\lambda \int_{\Omega} F(x, t \xi) d x \\
\leq & \frac{t^{p}}{p} \max \left\{\|\xi\|^{p},\|\xi\|^{q}\right\}-\frac{t^{1-\theta}}{1-\theta} \int_{\Omega_{1}} b(x)|\xi|^{1-\theta} d x .
\end{aligned}
$$

Since $p>1>1-\theta$, we have $\varphi_{\lambda}(t \xi)<0$ for $t<t_{2}$ with

$$
0<t_{2}<\min \left\{1,\left(\frac{p \int_{\Omega} b(x)|\xi|^{1-\theta} d x}{(1-\theta) \max \left\{\|\xi\|^{p},\|\xi\|^{q}\right\}}\right)^{1 /(p+\theta-1)}\right\}
$$

The proof of Step 2 is now complete.

Step 3. We show that there exists $u_{\lambda} \in E$ such that $\varphi_{\lambda}\left(u_{\lambda}\right)=$ $\inf _{u \in E} \varphi_{\lambda}(u)$ for any $\lambda \geq 0$.

Let $\left\{u_{\lambda}^{n}\right\} \subset E$ be a minimizing sequence of $\varphi_{\lambda}$. Then, using Step 1 , we get that $\left\{u_{\lambda}^{n}\right\}$ is a bounded sequence. So, there exists $u_{\lambda} \in E$ such that, up to a subsequence,

$$
\begin{gathered}
u_{\lambda}^{n} \rightarrow u_{\lambda} \quad \text { in } E, \\
u_{\lambda}^{n} \longrightarrow u_{\lambda} \quad \text { in } L^{p}(\Omega), \\
u_{\lambda}^{n}(x) \rightarrow u_{\lambda}(x) \quad \text { a.e.in } \Omega .
\end{gathered}
$$



that

Thus, as the proof of Step 3 in Theorem 1, we also obtain

$$
\begin{array}{r}
\lim _{n \rightarrow+\infty} \int_{\Omega} b(x)\left|u_{\lambda}^{n}\right|^{1-\theta} d x=\int_{\Omega} b(x)\left|u_{\lambda}\right|^{1-\theta} d x, \\
\lim _{n \rightarrow+\infty} \int_{\Omega} F\left(x, u_{\lambda}^{n}(x)\right)=\int_{\Omega} F\left(x, u_{\lambda}(x)\right) d x,
\end{array}
$$

and $\varphi_{\lambda}$ is weakly lower semicontinuous, and consequently,

$$
\varphi_{\lambda}\left(u_{\lambda}\right) \leq \liminf _{u \in E} \varphi_{\lambda}\left(u_{\lambda}^{n}\right)=\inf _{v \in E} \varphi_{\lambda}(v) \leq \varphi_{\lambda}\left(u_{\lambda}\right)
$$

which implies that

$$
\varphi\left(u_{\lambda}\right)=\inf _{u \in E} \varphi_{\lambda}\left(u_{\lambda}^{n}\right)
$$

The proof of Step 3 is complete.

Therefore, combining the above Steps 2 and 3, we deduce that $u_{\lambda}$ is the required nontrivial solution of problem $\left(P_{\lambda}\right)$. Thus, we complete the Proof of Theorem 2.

\section{Data Availability}

Data sharing is not applicable to this article as no new data were created or analyzed in this study.

\section{Conflicts of Interest}

The authors declare that they have no competing interests.

\section{Authors' Contributions}

The authors declare that the study was realized in collaboration with equal responsibility. All authors read and approved the final manuscript.

\section{Acknowledgments}

This work is supported by the National Key Research and Development Program of China (No. 2018YFC0310500), the Fundamental Research Funds for the Central Universities (No. 3072020CF2401), the Natural Science Foundation of Inner Mongolia (No. 2017MS0116), the National Natural Science Foundation of China (No. 11201095), the Postdoctoral Research Startup Foundation of Heilongjiang (No. LBHQ14044), and the Science Research Funds for Overseas Returned Chinese Scholars of Heilongiiang Province (No. LC201502).

\section{References}

[1] W. Liu and G. Dai, "Existence and multiplicity results for double phase problem," Journal of Differential Equations, vol. 265, no. 9, pp. 4311-4334, 2018.

[2] W. Liu and G. Dai, "Three ground state solutions for double phase problem," Journal of Mathematical Physics, vol. 59, no. 12, p. 121503, 2018.
[3] B. Ge and Z.-Y. Chen, "Existence of infinitely many solutions for double phase problem with sign-changing potential," Revista de la Real Academia de Ciencias Exactas, Físicas y Naturales. Serie A. Matemáticas, vol. 113, no. 4, pp. 3185-3196, 2019.

[4] B. Ge, D. J. Lv, and J. F. Lu, "Multiple solutions for a class of double phase problem without the Ambrosetti-Rabinowitz conditions," Nonlinear Analysis, vol. 188, pp. 294-315, 2019.

[5] B. Ge, L. Y. Wang, and J. F. Lu, "On a class of double-phase problem without Ambrosetti-Rabinowitz-type conditions," Applicable Analysis, vol. 2019, pp. 1-16, 2019.

[6] G. Hou, B. Ge, B. Zhang, and L. Wang, "Ground state signchanging solutions for a class of double phase problem in bounded domains," Boundary Value Problems, vol. 2020, no. 1,2020 .

[7] F. Colasuonno and M. Squassina, "Eigenvalues for double phase variational integrals," Annali di Matematica Pura ed Applicata, vol. 195, no. 6, pp. 1917-1959, 2016.

[8] K. Perera and M. Squassina, "Existence results for doublephase problems via Morse theory," Communications in Contemporary Mathematics, vol. 20, no. 2, article 1750023, 2017.

[9] Q. Zhang and V. D. Radulescu, "Double phase anisotropic variational problems and combined effects of reaction and absorption terms," Journal de Mathématiques Pures et Appliquées, vol. 118, pp. 159-203, 2018.

[10] X. Shi, V. D. Rădulescu, D. D. Repovš, and Q. Zhang, "Multiple solutions of double phase variational problems with variable exponent," Advances in Calculus of Variations, 2018.

[11] M. Cencelj, V. D. Rădulescu, and D. D. Repovš, "Double phase problems with variable growth," Nonlinear Analysis, vol. 177, pp. 270-287, 2018.

[12] V. V. Zhikov, "Averaging of functionals of the calculus of variations and elasticity theory," Izvestiya Akademii Nauk SSSR. Seriya Matematicheskaya, vol. 50, pp. 675-710, 1986.

[13] V. V. Zhikov, "On Lavrentiev's phenomenon," Russian Journal of Mathematical Physics, vol. 3, pp. 249-269, 1995.

[14] V. V. Zhikov, "On some variational problems," Russian Journal of Mathematical Physics, vol. 5, pp. 105-116, 1997.

[15] M. Colombo and G. Mingione, "Regularity for double phase variational problems," Archive for Rational Mechanics and Analysis, vol. 215, no. 2, pp. 443-496, 2015.

[16] M. Colombo and G. Mingione, "Bounded minimisers of double phase variational integrals," Archive for Rational Mechanics and Analysis, vol. 218, no. 1, pp. 219-273, 2015.

[17] P. Baroni, M. Colombo, and G. Mingione, "Regularity for general functionals with double phase," Calculus of Variations and Partial Differential Equations, vol. 57, no. 2, pp. 1-48, 2018.

[18] C. De Filippis and G. Mingione, "Manifold constrained nonuniformly elliptic problems," Journal of Geometric Analysis, vol. 30, no. 2, pp. 1661-1723, 2020.

[19] C. De Filippis and G. Mingione, "A borderline case of Calderon-Zygmund estimates for non-uniformly elliptic problems," Algebra i Analiz, vol. 31, pp. 82-115, 2019.

[20] P. Baroni, M. Colombo, and G. Mingione, "Harnack inequalities for double phase functionals," Nonlinear Analysis, vol. 121, pp. 206-222, 2015.

[21] S. S. Byun and J. Oh, "Global gradient estimates for the borderline case of double phase problems with BMO coefficients in nonsmooth domains," Journal of Differential Equations, vol. 263, no. 2, pp. 1643-1693, 2017. 
[22] J. Musielak, Orlicz Spaces and Modular Spaces, Lecture Notes in Math., vol. 1034, Springer, Berlin, 1983.

[23] A. Benkirane and M. Sidi El Vally, "Variational inequalities in Musielak-Orlicz-Sobolev spaces," Bulletin of the Belgian Mathematical Society, vol. 21, no. 5, pp. 787-811, 2014.

[24] X. Fan and C. X. Guan, "Uniform convexity of MusielakOrlicz-Sobolev spaces and applications," Nonlinear Analysis, vol. 73, no. 1, pp. 163-175, 2010.

[25] K. C. Chang, Critical Point Theory and Applications, Shanghai Scientific and thchnology Press, Shanghai, 1996. 